\title{
DNA Microarray Gene Expression Profile of Mycobacterium tuberculosis when Exposed to Osthole
}

\author{
JIAN WEI ${ }^{1,5 \dagger}, \mathrm{NA} \mathrm{GUO}^{2,4 \dagger}$, JUNCHAO LIANG ${ }^{2 \dagger}$, PENG YUAN ${ }^{2}$, QIYUN SHI ${ }^{2}, \mathrm{XUDONG} \mathrm{TANG}^{3 *}$ and LU YU ${ }^{2 *}$ \\ ${ }^{1}$ Life Science Department ChangChun Normal University, Changchun 130062, China \\ ${ }^{2}$ Key Laboratory for Zoonosis Research, Ministry of Education, Institute of Zoonosis, \\ College of Animal Science and Veterinary Medicine, Jilin University, Changchun 130062, P.R. China \\ ${ }^{3}$ Key Lab for New Drug Research of TCM, Research Institute of Tsinghua University in Shenzhen, \\ Shenzhen 518057, People's Republic of China \\ ${ }^{4}$ Department of Food Quality and Safety, College of Quartermaster Technology, Jilin University, \\ Changchun 130062, China \\ ${ }^{5}$ Bio-reactor Center, Jilin Agricultural University, Changchun, China
}

Received 21 July 2012, revised 12 November 2012, accepted 15 November 2012

\section{Abstract}

\begin{abstract}
Tuberculosis (TB), affecting one-third of the global population, kills an estimated two to three million people every year. The development of drug resistance is becoming a serious threat to any attempt to control this disease, which underscores the need for new agents targeting Mycobacterium tuberculosis (M. tuberculosis). Osthole (7-methoxy-8-isopentenoxycoumarin) is a coumarin derivative present in many medicinal plants. Previous studies have shown that osthole possesses antimycobacterial effects, however, the action mechanism of osthole is unclear. In the study, we used a commercial oligonucleotide microarray to determine the overall transcriptional response of M. tuberculosis $\mathrm{H} 37 \mathrm{Rv}$ triggered by exposure to osthole. Analysis of the microarray data revealed that a total of 478 genes were differentially regulated by osthole. Of these, 241 genes were upregulated, and 237 genes were downregulated. Some of the important genes that were significantly regulated are related to different pathways such as fumarate reductase, class I peroxidase, cell wall, nitrate respiration, and protein synthesis. Real-time quantitative RT-PCR was performed for chosen genes to validate the microarray results. To our knowledge, this genome-wide transcriptomics approach has produced the first insights into the response of $M$. tuberculosis when exposed to osthole.
\end{abstract}

Ke y words: Mycobacterium tuberculosis, antimycobacterial activity, microarray, osthole, transcriptome

\section{Introduction}

Mycobacterial diseases are still among the world's leading infection problems. The World Health Organization (WHO) estimates that nearly one third of the global population is infected with Mycobacterium tuberculosis (M. tuberculosis) (Raviglione, 2003). Pathogenic mycobacteria initiate long-term infection in the lungs by entering host macrophages and spreading rapidly. Whilst the currently available antimicrobials are often effective at eradicating the infection, there are issues with patient drug compliance and the emergence of drug-resistant M.tuberculosis (Ormerod 2005). In addition, the rising incidence of HIV infection, especially in parts of Africa, has further promoted mortality related to tuberculosis (Nunn et al., 2005). Although effective therapeutic regimens exist, the prevalence of M. tuberculosis strains resistant to antimicrobial agents for treatment of tuberculosis (TB) and the emergence of multidrug resistance urgently requires additional compounds targeted at new pathways.

Plants and other natural materials may prove to be valuable sources of useful new antimycobacterial drugs. Osthole (7-methoxy-8-isopentenoxycoumarin, chemical formula shown in Fig. 1), a coumarin derivative, has been extracted from many medicinal plants, such as Cnidium monnieri and Angelica pubescens. It is used in traditional Chinese medicine as tonics and aphrodisiacs. Previous studies have shown that osthole possesses antimycobacterial, antiproliferation, vasorelaxation,

* Corresponding author: Lu Yu, Key Laboratory of Zoonosis, Ministry of Education, Institute of Zoonosis, College of Animal Science and Veterinary Medicine, Jilin University, Changchun, 130062, P.R. China; phone: +86-431-87836713; fax: +86-431-87836160; e-mail: yulu225@126.com. Xudong Tang, Shenzhen High-Tech Industrial Estate, Nanshan, Shenzhen P.R. China. 10086. China; phone: + 86-755-26551339; fax: + 86-755-26957713; e-mail: tangxd@tsinghua-sz.org

$\dagger$ These authors should be regarded as joint First Authors. 


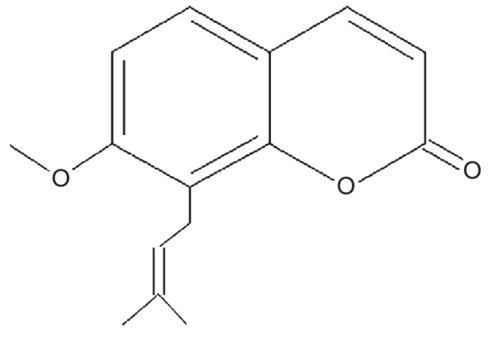

Fig. 1. Chemical formula of 7-methoxy-8-isopentenoxycoumarin.

antihepatitis, anti-inflammatory, antiaggregatory, and antiallergic effects (Figueroa et al., 2007; Huang et al., 1996; Liu et al., 1998; Matsuda et al., 2002; Yang et al., 2003). In our preliminary experiments, we found that osthole showed good antimycobacterial activity. However, few studies have reported on the mechanisms underlying the effects of antimycobacterial compounds (Betts et al., 2003).

DNA microarray technology has been used to understand biochemical pathways, to discover gene functions and to discover drug targets (Yu et al., 2007; Yu et al., 2008; Yu et al., 2010; Liang et al., 2011). Here, we describe the use of a microarray to generate the transcriptional response profile of M.tuberculosis following exposure to osthole to indicate the pathways induced by drug treatment, and a subset of the microarray results was verified by real-time RT-PCR.

\section{Experimental}

Materials and Methods

Bacterial strain and reagents. The M.tuberculosis strain H37Rv (American Type Culture Collection 27294) was obtained from the National Institute for the Control of Pharmaceutical and Biological Products. Middlebrook 7H9 broth and OADC (oleic acid, albumin, dextrose and catalase) were purchased from BD Biosciences, Inc., Sparks, MD. Alamar Blue was obtained from Trek Diagnostic Systems (Westlake, $\mathrm{OH}$, US). TRIzol was purchased from Invitrogen. Tween 80 was purchased from Sigma-Aldrich. Osthole was obtained from National Institute for the control of Pharmaceutical and biological products (Beijing, China). Stock solutions of osthole were prepared in dimethyl sulfoxide (DMSO, Sigma-Aldrich).

Antimycobacterial susceptibility test. The activity of osthole against the aforementioned $M$. tuberculosis strain was tested using a microplate Alamar Blue assay (MABA) according to the method of Franzblau et al. (Franzblau et al., 1998), as modified by JiménezArellanes et al (Jiménez-Arellanes et al., 2003). Briefly, sterile distilled water $(200 \mu \mathrm{l})$ was poured into the outer perimeter wells of the microplate. All other wells received $100 \mu \mathrm{l}$ of supplemented Middlebrook 7H9 broth. Then, working extract solutions $(100 \mu \mathrm{l})$ were poured into the first well of each row and two-fold dilution series were made from these solutions through the microplate column. The test inoculum $(100 \mu \mathrm{l})$ was added to all testing wells, as well as to the drug-free control wells. The final concentration of DMSO in the wells was approximately $1 \% \mathrm{v} / \mathrm{v}$. At the same time, controls diluted 10:100 and 1:100 were prepared from the bacterial suspension, representing the growth of $10 \%$ and $1 \%$ of the bacterial population tested, respectively. The final concentrations of osthole tested ranged from 1 to $512 \mu \mathrm{g} / \mathrm{ml}$. Each concentration was assayed in duplicate. Each microplate was incubated for five days at $37^{\circ} \mathrm{C}$ in a $5 \% \mathrm{CO}_{2}$ atmosphere in a sealed plastic bag. Following incubation, a control growth was developed with a mixture of $20 \mu$ of Alamar Blue solution (Trek Diagnostics, Westlake, $\mathrm{OH}$ ) and $12 \mu \mathrm{l}$ of sterile $10 \%$ Tween 80 . The plates were re-incubated at $37^{\circ} \mathrm{C}$ for $24 \mathrm{~h}$. After this incubation, if the well turned pink, all of the wells received a mixture of Alamar Blue and Tween solutions in the same way as described above and were incubated for an additional $24 \mathrm{~h}$. Wells with a well-defined pink color were scored as positive for growth. The minimal inhibitory concentration (MIC) was defined as the lowest concentration of a sample that prevents a color change to pink. Extracts were considered active if they showed an MIC $\leq 200 \mu \mathrm{g} / \mathrm{ml}$.

Cell culture and treatment with for microarray experiments. A frozen stock of $M$. tuberculosis strain H37Rv was inoculated into Middlebrook 7H9 broth containing $0.05 \%$ Tween $80,0.2 \%$ glycerol and $10 \%$ oleic acid, albumin, dextrose and catalase (OADC) and incubated at $37^{\circ} \mathrm{C}$ for five days. Then, the culture was transferred into $200 \mathrm{ml}$ of $7 \mathrm{H} 9$ media and incubated at $37^{\circ} \mathrm{C}$ with shaking until the $\mathrm{OD}_{600}$ reached 0.7 . Subsequently, $200 \mathrm{ml}$ of $7 \mathrm{H} 9$ broth was divided into two flasks, each of which contained $100 \mathrm{ml}$ of culture; the cells were harvested by centrifugation for RNA preparation. A osthole stock solution was prepared in dimethyl sulfoxide (DMSO). Drug treatment was conducted by adding the stock solution to one of the cultures to achieve a final concentration of $16 \mu \mathrm{g} / \mathrm{ml}(1 / 2 \mathrm{MIC})$. Untreated paired control bacteria were grown under identical conditions to treated bacteria, with the exception that no drug was added. The final concentration of DMSO in each culture could not exceed $0.05 \%(\mathrm{v} / \mathrm{v})$ (Slayden et al., 2006).

Two independent $200 \mathrm{ml}$ cultures were prepared to act as biological repeats. Upon completion of the predefined duration $(4 \mathrm{~h})$ of drug and control treatments, the bacteria were harvested by centrifugation and then stored for RNA extraction. 
RNA isolation and cDNA labeling. Bacterial cultures were centrifuged for $5 \mathrm{~min}$ at $2500 \mathrm{~g}$. After removing the supernatant, the pellets were frozen on dry ice and stored at $-80^{\circ} \mathrm{C}$. Total RNA was harvested using TRIzol (Invitrogen) and an RNeasy kit (Qiagen) according to the manufacturer's instructions, including a DNase digestion step. The RNA samples were redissolved to produce a final concentration of $300-500 \mathrm{ng} / \mu \mathrm{l}$. For every RNA sample, $120 \mu \mathrm{l}$ was sent to Shanghai Bio Co., Ltd. and further examined through a quality and quantity test based on electrophoresis before microarray hybridization.

Fluorescently labeled cRNA, transcribed from cDNA, was produced using a Quick Amp Kit, PLUS, Two-Color (Agilent p/n 5190-0444) in Agilent's SureHyb Hybridization Chambers. The cRNA was labeled with the fluorescent dyes Cy5 and Cy3-CTP. Double-stranded cDNA was synthesized from $1 \mu \mathrm{g}$ of total RNA using a cDNA synthesis kit according to the manufacturer's protocol (Quick Amp Kit, Agilent). T7 promoter primers were used instead of the poly-T primer provided in the kit. The Cy3- and Cy5-labeled products were purified using an RNeasy Mini Kit (Qiagen). An aliquot of $1 \mu \mathrm{l}$ of purified cRNA was used to determine the yield and specific activity with a NanoDrop ND-1000. The amount of Cy3- or Cy5-labeled cRNA was determined by measuring the absorbance at A260 nm, A280 nm, A550 nm (Cy3) and A650 (Cy5). The specific activity (pmol dye per $\mu \mathrm{g}$ cRNA) of the cRNA can be obtained from the following calculation: specific activity $=($ concentration of $\mathrm{Cy} 3 / \mathrm{Cy} 5) /\left((\right.$ concentration of $\left.\mathrm{CRNA}){ }^{\star} 1000\right)=\mathrm{pmol}$ Cy3/Cy5 per $\mu \mathrm{g}$ cRNA. If the yield is $<825 \mathrm{ng}$ and the specific activity is $<8.0 \mathrm{pmol}$ Cy $3 / \mathrm{Cy} 5$ per $\mu \mathrm{g}$ of cRNA, the experiment does not proceed to the hybridization step. cRNA was repeatedly prepared.

Microarray hybridization and analysis. M. tuberculosis microarray slides consisted of 4690 60-mer oligonucleotides representing 4004 open reading frames from M. tuberculosis strain H37Rv and 686 unique open reading frames from strain CDC1551 that are not present in the H37Rv strain's annotated gene complement. Microarray hybridization was performed in Agilent's SureHyb Hybridization Chambers using the Agilent Gene Expression Hybridization Kit. After hybridization and washing, the processed slides were scanned using an Agilent DNA microarray scanner (part number G2505B) with the settings recommended by Agilent Technologies. The resulting text files, which were extracted using Agilent Feature Extraction Software (version 10.5.1.1), were imported into Agilent GeneSpring GX software (version 11.0) for further analysis. The microarray datasets were normalized in Agilent Feature Extraction Software (mainly LOWESS normalization) and then genes marked as present were chosen for further analysis. Differentially expressed genes were identified through Volcano Plot screening. Cluster analysis was carried out by hierarchical clustering (HCL). In addition to the significance analysis of the microarrays, a fold change analysis was performed in which the ratios of the geometric means of the expression intensities of the corresponding genes in the osthole treatment samples relative to control samples were calculated. The ratios were reported as the -fold change up or down. To select differentially expressed genes, the genes were considered to be significantly differentially hybridized compared with the M. tuberculosis control if they displayed at least a twofold difference in the ratio (Frota et al., 2004).

Quantitative real-time reverse transcription RTPCR. Aliquots of the RNA preparations from the osthole-treated and control samples used in the microarray experiments were saved for follow-up quantitative real-time RT-PCR. Quantitative real-time RT-PCRs were performed in triplicate using the 7000 Sequence Detection System (Applied Biosystems, Foster City, CA) according to a previously described procedure (Yu et al., 2007). The primer sequences used are listed in Table I.

\section{Results}

Gene transcription responses to osthole exposure. In this experiment, the MIC value of osthole against M. tuberculosis H37Rv (ATCC 27294) was $32 \mu \mathrm{g} / \mathrm{ml}$. Whole-genome microarrays were employed to analyze gene expression in $M$. tuberculosis $\mathrm{H} 37 \mathrm{Rv}$ when exposed to a subinhibitory (1/2 MIC) concentration of osthole for $4 \mathrm{~h}$. Overall, there were 478 genes differentially regulated by osthole. Among these, 241 exhibited a significant increase in traIscription and 237 exhibited a significant decrease in transcription. The microarray-related data were submitted to Gene Expression Omnibus (GEO) under accession number GSE32076. The genes which were differentially expressed in response to osthole and their biological roles according to their functional class are shown in the supplementary material attached to PJM Vol. 62, No 1 (Table SI on line at http:// www.pjm.microbiology.pl), and some genes which are high differently expressed in response to osthole were listed in Table II.

Of the genes that showed a significant response to osthole, most were classified as having an unclassified role category not yet assigned (24.7\%) and the others were classified as involved in biosynthesis of cofactors, prosthetic groups, and carriers (2.1\%), cell envelope (5.6\%), cellular processes $(2.1 \%)$, central intermediary metabolism (4.4\%), conserved hypothetical proteins (14.2\%), DNA metabolism (2.3\%), energy metabolism (6.3\%), hypothetical proteins $(13.0 \%)$, protein synthesis $(2.7 \%)$, regulatory functions $(5.0 \%)$, transport and binding proteins $(4.2 \%)$, unknown function $(7.1 \%)$ 
Table I

Primers used in real-time RT-PCR with SYBR green probes.

\begin{tabular}{|l|l|l|l|}
\hline \multicolumn{1}{|c|}{ Primer } & Systematic name & \multicolumn{1}{c|}{ Sequence ( ${ }^{\prime}$-3') } & \multicolumn{1}{c|}{ Reference } \\
\hline 16S rRNAfor & & GCACCGGCCAACTACGTG & Grassi et al. \\
\hline 16S rRNArev & & GAACAACGCGACAAACCACC & Grassi et al. \\
\hline$m m p S 5$ for & Rv0677c & CAAGGTGGTGGAGTACGAAGTTT & This study \\
\hline$m m p S 5$ rev & Rv0677c & TCGAGGTCCAGGTAGTTGATGTTG & This study \\
\hline infC for & Rv1641 & AGACCGTCGTCAAAGAACAAAAG & This study \\
\hline infC rev & Rv1641 & TTGGTCTCGTAATCGTGATCGT & This study \\
\hline nirA for & Rv2391 & CTGGACCGGTGACGACAAC & Liang J et al. \\
\hline nirA rev & Rv2391 & CAACGCACCCGCATCA & Liang J et al. \\
\hline cysH for & Rv2392 & TGCGATCGAGTCCGTCTATG & Liang J et al. \\
\hline cysH rev & Rv2392 & TGTGTGCTCCGGAGTGACAT & Liang J et al. \\
\hline tgs 1 for & Rv3130c & GATTCGCTGCGTACGCTAGTG & This study \\
\hline tgs 1 rev & Rv3130c & ACACGGTTATCGGTCTTGCTC & This study \\
\hline$s i g E$ for & Rv1221 & TCCGGTCGGTCCAGAATTAC & This study \\
\hline sigE rev & Rv1221 & TTGGTGGTGATGCGGTGTAG & This study \\
\hline groEL2 for & Rv0440 & TCGAGGAGTCCAACACCTTTG & This study \\
\hline groEL2 rev & Rv0440 & TCACGAAGTACCCCGAGATGTA & This study \\
\hline$m p t 83$ for & Rv2873 & TTGGTATGTGGCGGAGTTCA & This study \\
\hline$m p t 83$ rev & Rv2873 & AGCACCGTATCGATCATGTA & This study \\
\hline frdD for & Rv1555 & GCAACCCGATCACCAAGCTTGTT & This study \\
\hline frdD rev & Rv1555 & CATGGTCGAGCACGAACCGGAAC & This study \\
\hline frdA for & Rv1552 & ACGAGCACAACAAAGGAAGGA & This study \\
\hline frdA rev & Rv1552 & AGGTGCCGCAGGTCTAGATAGA & This study \\
\hline cadI for & Rv2641 & GAGGCCGCAATCACGTTCTA & This study \\
\hline cadI rev & Rv2641 & TATCCGGGCTTGCGTTTG & This study \\
\hline
\end{tabular}

Table II

Selected genes which are high differently expressed in response to osthole.

\begin{tabular}{|l|l|l|l|c|}
\hline \multicolumn{1}{|c|}{ Functional category } & $\begin{array}{c}\text { Systematic } \\
\text { name }\end{array}$ & Gene & \multicolumn{1}{|c|}{ Product or putative function } & $\begin{array}{c}\text { Fold } \\
\text { change }\end{array}$ \\
\hline $\begin{array}{l}\text { Biosynthesis of cofactors, } \\
\text { prosthetic groups, and carriers }\end{array}$ & Rv3398c & idsA1 & $\begin{array}{l}\text { Multifunctional dimethylallyltransferase \% 2Ffarnesyl } \\
\text { diphosphate synthetase \% 2F farnesyltranstransferase }\end{array}$ & 3.96 \\
\hline Cell envelope & Rv0676c & mmpL5 & Transmembrane transport protein mmpl5 & 9.98 \\
\cline { 2 - 6 } & Rv0450c & mmpL4 & Transmembrane transport protein mmpl4 & 3.06 \\
\hline Central intermediary metabolism & Rv3397c & phyA & Phytoene synthase & 4.16 \\
\hline Energy metabolism & Rv0136 & cyp138 & Cytochrome P450 138 & 6.38 \\
\cline { 2 - 6 } & Rv2391 & nirA & Ferredoxin-dependent nitrite reductase NIRA & 3.03 \\
\hline Protein fate & Rv3418c & groES & Co-chaperonin groes & -3.06 \\
\cline { 2 - 5 } & Rv3417c & groEL & Chaperonin groel & -3.06 \\
\hline \multirow{2}{*}{ Regulatory functions } & Rv0135c & - & Transcriptional regulatory protein & 4.22 \\
\cline { 2 - 5 } & Rv0452 & - & Transcriptional regulatory protein & 3.97 \\
\cline { 2 - 5 } & Rv1685c & - & Hypothetical protein & 10.28 \\
\hline Transport and binding proteins & Rv1686c & - & Integral membrane protein ABC transporter & 13.20 \\
\hline
\end{tabular}

"+" increase and "_" reduction

and other categories (6.3\%) as shown in Fig. 2. In this study, our interest was focused mainly on specific genes that could affect the organism's survival when exposed to osthole, and these were mainly involved in different pathways, such as fumarate reductase, class I peroxidase, nitrate respiration, and genes involved in the cell wall and protein synthesis. These genes will be discussed in the "Discussion" section. 


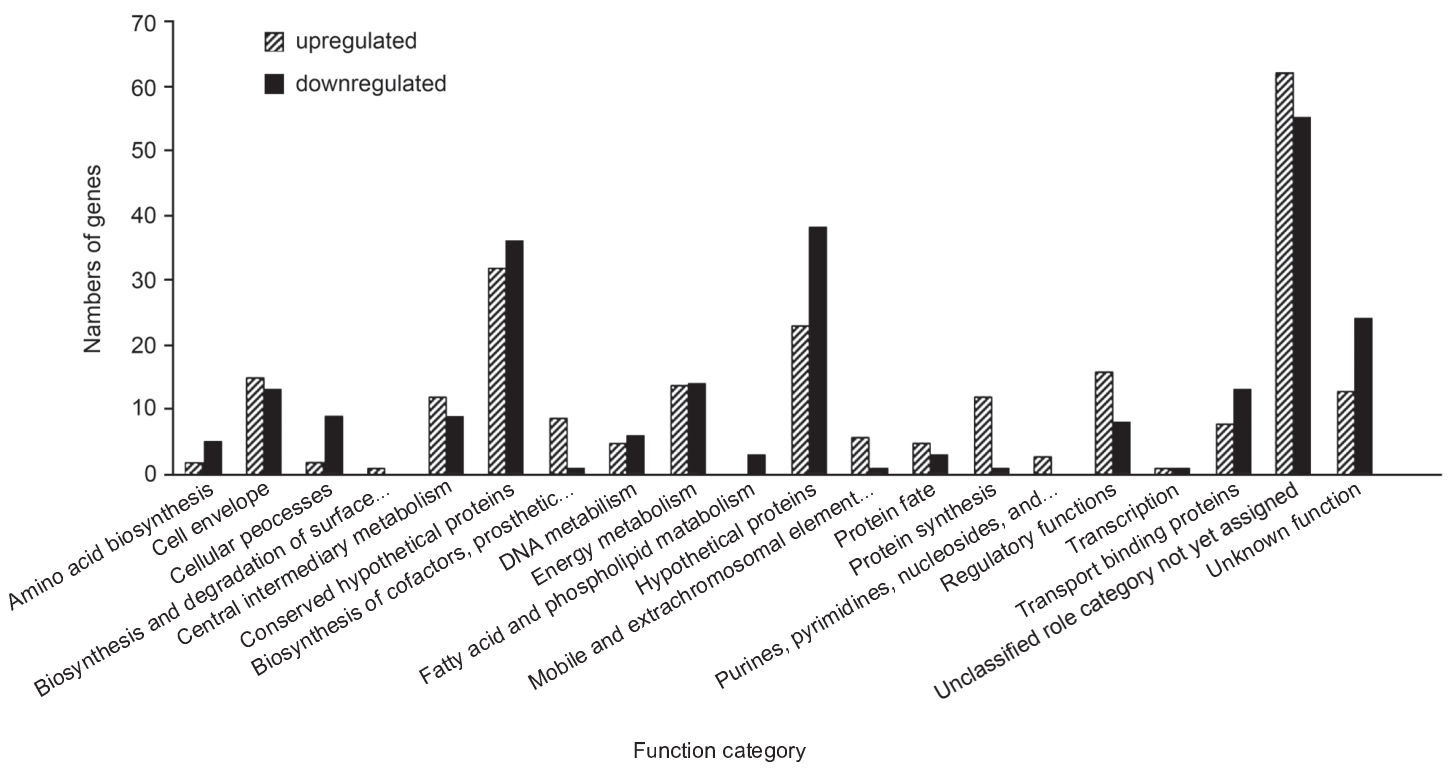

Fig. 2. The genes that showed a significant response to osthole.

Validating microarray data by real-time RT-PCR. Eleven genes were selected (i.e. $m m p S 5$, infC, nirA, cys $H$, tgs 1, sigE, groEL2, mpt83, frdD, frdA and cadI) to verify the microarray data by the technique of realtime quantitative RT-PCR. In summary, there was a good correlation between the microarray data and the real-time RT-PCR data (Table III). Among the eleven genes, six genes exhibited upregulation and five genes exhibited downregulation upon exposure to osthole. It is notable that for two genes, mmpS5 and groEL2 the real-time RT-PCR results showed greater $\mathrm{n}$-fold changes than the microarray results, which may be reflective of the greater dynamic range of real-time RTPCR analysis. For nine genes (infC, nirA, cysH, tgsl, sigE, $m p t 83, f r d D, f r d A$ and $c a d I)$, the expression levels did not differ markedly between the real-time RT-PCR data and the microarray data.

\section{Discussion}

Inhibition of the fumarate reductase genes in the presence of osthole. In our study, the genes $f r d A$, $f r d B, f r d C$ and $f r d D$ encoding fumarate reductase were

Table III

Real-time RT-PCR analysis of gene expression.

\begin{tabular}{|c|c|c|c|c|}
\hline \multirow{2}{*}{$\begin{array}{c}\text { Systematic } \\
\text { name }\end{array}$} & \multirow{2}{*}{ Gene } & \multirow{2}{*}{ Description } & \multicolumn{2}{|c|}{ Fold change } \\
\hline & & & RT-PCR & Microarray \\
\hline Rv0677c & mmpS5 & hypothetical protein & +78.03 & +16.90 \\
\hline Rv1641 & $\operatorname{infC}$ & translation initiation factor IF-3 & +5.83 & +2.55 \\
\hline Rv2391 & nirA & ferredoxin-dependent nitrite reductase NIRA & +2.31 & +3.03 \\
\hline Rv2392 & cysH & phosphoadenosine phosphosulfate reductase & +3.08 & +2.72 \\
\hline Rv3130c & $\operatorname{tgs} 1$ & triacylglycerol synthase & +3.64 & +2.53 \\
\hline Rv1221 & sigE & RNA polymerase sigma factor & +2.64 & +2.31 \\
\hline Rv0440 & groEL2 & chaperonin GroEL & -19.35 & -4.29 \\
\hline Rv2873 & $m p t 83$ & cell surface lipoprotein mpt83 \%28lipoprotein P23\%29 & -2.12 & -2.45 \\
\hline Rv1555 & $f r d D$ & fumarate reductase subunit D & -3.01 & -2.23 \\
\hline Rv1552 & frdA & fumarate reductase subunit $\mathrm{A}$ & -4.11 & -2.16 \\
\hline Rv2641 & cadI & cadmium inducible protein & -2.38 & -2.03 \\
\hline
\end{tabular}

“+” increase and "_" reduction. 
downregulated by 2.16-fold, 2.06-fold, 2.11-fold and 2.23 -fold respetively when exposed to osthole. Fumarate reductase (FRD) is an important enzyme in fumarate respiration, which is an alternative means of acquiring energy by utilizing fumarate as the terminal electron acceptor when oxygen or nitrate is absent. As previously reported, $f r d A$ has been found to be upregulated in $M$. tuberculosis interacting with the macrophage versus M. tuberculosis grown in broth cultures (Schnappinger et al., 2003) as well as in studies that examined the behaviour of M. tuberculosis grown under carbon starvation (Betts etal., 2002). The transcriptome in stationary-phase M.tuberculosis $\mathrm{H} 37 \mathrm{Rv}$ cultures was found to increase $f r d B$ and $f r d C$ transcripts versus bacteria in exponential growth (Raman et al., 2001). Additionally, investigations into the respiratory behaviour of Mycobacterium phlei found that FRD activity increased fourfold when bacteria were grown under low-oxygen conditions (Gillespie et al., 1988). With regard to other pathogenic organisms, FRD has been considered a target in the treatment of Helicobacter pylori, as it was found to be an essential gene in the establishment of $H$. pylori colonization of the mouse stomach (Wang et al., 2000). Furthermore, FRD has been reported to be a successful target in the treatment of protozoan and helminth infections using a variety of compounds (Chen et al., 2001; Omura et al., 2001). Our results suggest the FRD may be a novel target in $M$. tuberculosis when exposed to osthole and the mechanism involved needs further study.

Genes involved in nitrate respiration were inhibited when exposed to osthole. Nitrate respiration is an important physiological process that allows bacteria to generate sufficient energy to permit anaerobic growth. Lastly, nitrate respiration was found equally important in protecting $M$. tuberculosis against reactive nitrogen species which are likely to be encountered by the pathogen in the infected host (Tan et al., 2010). During nitrate respiration, nitrate is reduced to nitrite in the cytoplasm by a membrane-bound nitrate reductase (Nishimura et al., 2008). Nitrate reductase, encoded by narGHJI, is a membrane-bound molybdenum-containing complex and is typical for a respiratory enzyme (Malm et al., 2009). The membrane-bound nitrate reductase is a three-subunit protein, with the transmembrane $\gamma$ subunit (NarI) anchoring the cytoplasmically exposed a subunit (NarG) and $\beta$ subunit ( $\mathrm{NarH}$ ) to the membrane. In our study, on exposure to osthole, the genes narJ, narI, narH and narG were all downregulated by 2.08-fold, 2.28-fold, 2.20-fold, and 2.15-fold, respectively. The expression of narKGHJI operon is regulated by anaerobiosis and nitrate (Nishimura et al., 2007).

Assimilation of nitrogen into mycobacterial metabolism is essential for the survival of M. tuberculosis in vitro and in vivo (Malm et al., 2009). It is suggested that narGHJI mediates nitrate assimilation in M. tuberculosis
(Malm et al., 2009). Though nitrate can not be utilized as a nitrogen source, $M$. tuberculosis can utilize nitrite as a source of nitrogen for growth. The nar $G$ mutant showed no nitrate reductase activity in whole culture or in cell-free assays (Sohaskey and Wayne, 2003). In the absence of the NarJ protein, the balanced overproduction of subunits $\alpha, \beta$ and $\gamma$ from a multicopy plasmid does not result in the formation of either fully active $\alpha-\beta$ or $\alpha-\beta-\gamma$ complex (Dubourdieu and De Moss, 1992). The downregulation of the genes may inhibit the activity and biosynthesis of nitrate reductase, which affect the nitrate respiration and attenuate the ability of assimilation of nitrogen.

Increased transcription of the gene cluster related to the biosythesis of polyacyltrehalose. In the study, the genes pks3, papA3 and $m m p L 10$ were upregulated by 2.34 -fold, 2.06-fold and 2.27-fold, respectively, when exposed to osthole. These genes reside in the gene cluster pks3/4-papA3-mmpL10, which is involved in the biosynthesis of polyacyltrehalose (PAT). PAT, a pentaacylated, trehalose-based glycolipid, is a cell wall molecule unique to pathogenic M. tuberculosis. In M. tuberculosis, PapA3 encoded by papA3 is an acyltransferase essential for the biosynthesis of PAT and was selective for trehalose; no activity was observed with other structurally related disaccharides. Disruption of the papA3 gene from $M$. tuberculosis resulted in the loss of PAT from bacterial lipid extracts (Hatzios et al., 2009). Pks3 is a polyketide synthase, which is involved in the synthesis of mycolipanolic and mycolipenic acids, the trimethyl branched fatty acids present in the PAT of M. tuberculosis (Matsuda et al., 2002). In some strains, including the sequenced $\mathrm{H} 37 \mathrm{Rv}$ strain, there is an intervening stop codon in $p k s 3 / 4$ that results in two separate open reading frames (termed $p k s 3$ and $p k s 4$ ) (Dubey et al., 2002). Strains containing this mutation do not synthesize PAT (Domenech et al., 2005), indicating that an intact $p k s 3 / 4$ gene is essential for the biosynthesis of this glycolipid. Within the same gene cluster resides $m m p L 10$ encoding a putative lipid transporter, which transports PAT or its precursor to the cell surface. Previous studies reported that the genes from the PAT biosynthetic gene cluster were upregulated under various conditions of environmental stress, including phagosomal acidification and nutrient starvation (Rohde et al., 2007). This region was also upregulated in M. bovis after acid shock, reaching maximum expression after $24 \mathrm{~h}$ (Golby et al. 2007). Hence, the upregulation of the genes may be an adaptive response to environmental changes for survival of the organism. The trigger for the induced transcription of the gene cluster in M. tuberculosis following osthole challenge requires further study.

Upregulation of genes involved in the ribosome proteins in osthole. Following exposure to osthole, the genes $\mathrm{rpsH}$ and $\mathrm{rpsS}$ encoding $30 \mathrm{~S}$ ribosome proteins 
were upregulated by 2.03-fold and 2.28-fold; the genes encoding $50 \mathrm{~S}$ ribosomal proteins were also upregulated from 2.03-fold to 2.21-fold, including $r p l W, r p l T$, $r p l O, r p l D$ and $r p l B$. The initiation factors infC, which is essential for the initiation of translation, was upregulated by 2.55 -fold and the levels of expression of the gene rho, which encodes transcription termination factor, was also increased by 2.06 -fold. A recent study showed that exposure of Clostridium difficile to three subinhibitory concentrations of antibiotics (amoxicillin, clindamycin, and metronidazole) resulted in a general upregulation of the transcription and translation machinery, including significant upregulation of 25 ribosomal protein-encoding genes (in addition to four located elsewhere) (Emerson et al., 2008). Moreover, Dennis demonstrated that when experiencing low levels of translation inhibition, cells can compensate by upregulation of the synthesis of ribosomal proteins, but as inhibition increases, this compensation fails to keep pace (Dennis, 1976). In the study, these genes encode essential components of the protein synthesis pathway. Their upregulation may be a response to the translation inhibition caused by osthole targeting the ribosome or its associated translation factors, which aims at surviving antimicrobial challenge.

The downregulation of the genes related to arginine biosynthesis. In many microorganisms, L-arginine is used as a source of both carbon and nitrogen, and the transport and metabolism of L-arginine has been shown to be essential for Mycobacterium tuberculosis (Gordhan et al., 2002). In the study, the genes $\operatorname{argR,} \arg , \arg G, \arg F$ and $\arg D$, residing in the arginine biosynthetic gene cluster $(\operatorname{argCJBDFRGH})$, were inhibited by 2.37 -fold, 2.37-fold, 2.47-fold, 2.36-fold and 2.38-fold respectively. As a previous study reported, deletion of gene $\arg R$ in Legionella pneumophila does affect the transcript level of genes predicted to encode the terminal steps of L-arginine biosynthesis from L-ornithine (Hovel-Miner et al., 2010). Interestingly, the mutant of Streptomyces clavuligerus with an argJ disruption is auxotrophic, which was suggested to be a polar effect on the transcription of arginine biosynthesis genes located downstream of argJ (de la Fuente et al., 2004). In M. tuberculosis, the $\arg F$ mutant strains required exogenous $\mathrm{L}$-arginine for growth in vitro and were defective in $\arg G$ function. The mutant strains exerted polar effects on $\arg R$ and $\arg G$, and also exhibited a very strong attenuation in virulence, which was reflected in a reduction in c.f.u. values and survival in immunocompetent mice (Gordhan etal., 2002). In Listeria monocytogenes, the insertional mutation of $\operatorname{argD}$ led to a reduced replication rate in Caco-2 cells (Joseph et al., 2006). Moreover, in Corynebacterium glutamicum, genes encoding proteins of the L-arginine biosynthesis pathway ( $\arg B, \arg C, \arg D, \operatorname{argF}, \arg G$, $\operatorname{argH}, \operatorname{argJ}, \operatorname{argR}$ and $\operatorname{argS}$ ) showed a decreased expres- sion in ammonium-limited chemostat cultures (Silberbach et al., 2005).

Hence, the downregulation of the genes may affect the biosynthesis of the L-arginine, which destroyed the survival ability of $M$. tuberculosis in the presence of osthole.

These results show that osthole has potential antimycobacterial activity. Our DNA microarray analysis demonstrated that osthole affected a number of important genes involved in different metabolic pathways in M. tuberculosis. These findings will pave the way for exploring the responsive mechanisms of $M$. tuberculosis to osthole treatment.

\section{Acknowledgements}

Financial supports for this work came from National Nature Science Foundation of China (No. 31000822); the Specialized Research Fund for Important National Science \& Technology Specific Projects (2012ZX10003002); National Nature Science Foundation of China (No. 31172364); New Century Excellent Talents in University of Ministry of Education of China (2009 year); Shenzhen Biological Special Funds for Industrial Development Aid Key Basic Research Project (JC201005280643A); Shenzhen Special Funds for Key Laboratory Construction Aid Project (SW201110026).

\section{Literature}

Barry. 2004. The transcriptional responses of Mycobacterium tuberculosis to inhibitors of metabolism: novel insights into drug mechanisms of action. J. Biol. Chem. 279: 40174-40184.

Betts J.C., A. McLaren, M.G. Lennon, F.M. Kelly, P.T. Lukey, S.J. Blakemore and K. Duncan. 2003. Signature gene expression profiles discriminate between isoniazid-, thiolactomycin-, and triclosan-treated Mycobacterium tuberculosis. Antimicrob. Agents. Chemother. 47: 2903-2913.

Betts J.C., P.T. Lukey, L.C. Robb, R.A. McAdam and K. Duncan. 2002. Evaluation of a nutrient starvation model of Mycobacterium tuberculosis persistence by gene and protein expression profiling. Mol. Microbiol. 43: 717-731.

Chen M, L. Zhai, S.B. Christensen, T.G. Theander and A. Kharazmi. 2001. Inhibition of fumarate reductase in Leishmania major and $L$. donovani by chalcones. Antimicrob. Agents. Chemother. 45: 2023-2029.

Dennis P.P. 1976. Effects of chloramphenicol on the transcriptional activities of ribosomal RNA and ribosomal protein genes in Escherichia coli. J. Mol. Biol.108: 535-546.

Dubey V.S., T.D. Sirakova and P.E. Kolattukudy. 2002. Disruption of $m s l 3$ abolishes the synthesis of mycolipanoic and mycolipenic acids required for polyacyltrehalose synthesis in Mycobacterium tuberculosis $\mathrm{H} 37 \mathrm{Rv}$ and causes cell aggregation. Mol. Microbiol. 45: 1451-1459.

Dubourdieu, M. and J.A. DeMoss. 1992. The narJ gene product is required for biogenesis of respiratory nitrate reductase in Escherichia coli. J. Bacteriol. 174: 867-872.

de la Fuente A., J.F. Martín, A. Rodríguez-García and P. Liras. 2004. Two proteins with ornithine acetyltransferase activity show different functions in Streptomyces clavuligerus: Oat2 modulates clavulanic acid biosynthesis in response to arginine. J. Bacteriol. 186: 6501-6507.

Domenech P., M.B. Reed and C.E. 3rd. Barry. 2005. Contribution of the Mycobacterium tuberculosis MmpL protein family to virulence and drug resistance. Infect. Immun. 73: 3492-3501. 
Emerson J.E., R.A. Stabler, B.W. Wren and N.F. Fairweather. 2008. Microarray analysis of the transcriptional responses of Clostridium difficile to environmental and antibiotic stress. J. Med. Microbiol. 57: 757-764

Figueroa M., I. Rivero-Cruz, B. Rivero-Cruz, R. Bye, A. Navarrete and R. Mata. 2007.Constituents, biological activities and quality control parameters of the crude extract and essential oil from Arracacia tolucensis var. Multifida. J. Ethnopharmacol. 113: 125-131.

Franzblau S.G., R.S. Witzig, J.C. McLaughlin, P. Torres, G. Madico, A. Hernandez, M.T. Degnan, M.B. Cook, V.K. Quenzer, R.M. Ferguson and others. 1998. Rapid low-technology MIC determination with clinical, Mycobacterium tuberculosis isolates by using the microplate Alamar Blue assay. J. Clin. Microbiol. 36: 362-366.

Frota C.C., D.M. Hunt, R.S. Buxton, L. Rickman, J. Hinds, K. Kremer, D. van Soolingen and M.J. Colston. 2004. Genome structure in the vole bacillus, Mycobacterium microti, a member of the Mycobacterium tuberculosis complex with a low virulence for humans. Microbiology 150: 1519-1527.

Gillespie J., L.L. Barton and E.W. Rypka. 1988. Influence of oxygen tension on the respiratory activity of Mycobacterium phlei. J. Gen. Microbiol. 134: 247-252.

Golby P., K.A. Hatch, J. Bacon, R. Cooney, P. Riley, J. Allnutt, J. Hinds, J. Nunez, P.D. Marsh, R.G. Hewinson and others. 2007. Comparative transcriptomics reveals key gene expression differences between the human and bovine pathogens of the Mycobacterium tuberculosis complex. Microbiology 153: 3323-3336.

Gordhan B.G., D.A. Smith, H. Alderton, R.A. McAdam, G.J. Bancroft and V. Mizrahi. 2002. Construction and phenotypic characterization of an auxotrophic mutant of Mycobacterium tuberculosis defective in 1-Arginine biosynthesis. Infect. Immun. 70: 3080-3084. Grassi M., E. Volpe, V. Colizzi and F. Mariani. 2006. An improved, real-time PCR assay for the detection of GC-rich and low abundance templates of Mycobacterium tuberculosis. J. Microbiol. Methods 64: 406-410.

Hatzios S.K., M.W. Schelle, C.M. Holsclaw, C.R. Behrens, Z. Botyanszki, F.L. Lin, B.L. Carlson, P. Kumar, J.A. Leary and C.R. Bertozzi. 2009. PapA3 is an acyltransferase required for polyacyltrehalose biosynthesis in Mycobacterium tuberculosis. J. Biol. Chem. 284: 12745-12751.

Hovel-Miner G., S.P. Faucher, X. Charpentier and H.A. Shuman. 2010. ArgR-regulated genes are derepressed in the legionella-containing vacuole. J. Bacteriol. 192: 4504-4516.

Huang R.L., C.C. Chen, Y.L. Huang, D.J. Hsieh, C.P. Hu, C.F. Chen and C. Chang. 1996. Osthole increases glycosylation of hepatitis B surface antigen and suppresses the secretion of hepatitis B virus in vitro. Hepatology 24: 508-515.

Jiménez-Arellanes A., M. Meckes, R. Ramírez, J. Torres and J. LunaHerrera. 2003. Activity against multidrug-resistant Mycobacterium tuberculosis in Mexican plants used to treat respiratory diseases. Phytother. Res. 17: 903-908.

Joseph B., K. Przybilla, C. Stïhler, K. Schauer, J. Slaghuis, T.M. Fuchs, W. Goebel. 2006. Identification of Listeria monocytogenes genes contributing to intracellular replication by expression profiling and mutant screening. J. Bacteriol. 188: 556-568.

Liang J., F. Zeng, A. Guo, L. Liu, N. Guo, L. Li, J. Jin, X. Wu, M. Liu, D. Zhao, Y. Li, Q. Jin and L. Yu, 2011. Microarray analysis of the chelerythrine-induced transcriptome of Mycobacterium tuberculosis. Curr. Microbiol. 62: 1200-1208.

Liu J.H., S. Zschocke, E. Reininger and R. Bauer. 1998. Inhibitory effects of Angelica pubescens f. biserrata on 5-lipoxygenase and cyclooxygenase. Planta. Med. 64: 525-529.

Malm S., Y. Tiffert, J. Micklinghoff, S. Schultze, I. Joost, I. Weber, S. Horst, B. Ackermann, M. Schmidt, W. Wohlleben and others. 2009. The roles of the nitrate reductase NarGHJI, the nitrite reductase NirB D and the response regulator GlnR in nitrate assimilation of Mycobacterium tuberculosis. Microbiology 155: 1332-1339.
Matsuda H., N. Tomohiro, Y. Ido and M. Kubo. 2002. Anti-allergic effects of cnidii monnieri fructus (dried fruits of Cnidium monnieri) and its major component, osthol. Biol. Pharm. Bull. 25: 809-812. Nishimura T., H. Teramoto, A.A. Vertès, M. Inui and H. Yukawa. 2008. ArnR, a novel transcriptional regulator, represses expression of the narKGHJI operon in corynebacterium glutamicum. J. Biochem. 190: 3264-3273.

Nishimura T., A.A. Vertès, Y. Shinoda, M. Inui, H. Yukawa. 2007. Anaerobic growth of Corynebacterium glutamicum using nitrate as a terminal electron acceptor. Appl. Microbiol. Biotechnol. 75: 889-897. Nunn P., B. Williams, K. Floyd, C. Dye, G. Elzinga and M. Raviglione. 2005. Tuberculosis control in the era of HIV. Nat. Rev. Immunol. 5: 819-826.

Omura S., H. Miyadera, H. Ui, K. Shiomi, Y. Yamaguchi, R. Masama, T. Nagamitsu, D. Takano, T. Sunazuka, A. Harder and others. 2001. An anthelmintic compound, nafuredin, shows selective inhibition of complex I in helminth mitochondria. Proc. Natl. Acad. Sci. USA 98: 60-62.

Ormerod L.P. 2005. Multidrug-resistant tuberculosis (MDR-TB): epidemiology, prevention and treatment. Br. Med. Bull. 17-24. Raman S., T. Song, X. Puyang, S. Bardarov, W.R. Jr. Jacobs and R.N. Husson. 2001. The alternative sigma factor SigH regulates major components of oxidative and heat stress responses in $\mathrm{Myco}$ bacterium tuberculosis. J. Bacteriol. 183: 6119-6125.

Raviglione M.C. 2003. The TB epidemic from 1992 to 2002. Tuberculosis (Edinb) 83: 4-14.

Rohde K.H., R.B. Abramovitch and D.G. Russell. 2007. Mycobac terium tuberculosis invasion of macrophages: linking bacterial gene expression to environmental cues. Cell Host Microbe. 2: 352-364. Schnappinger D., S. Ehrt, M.I. Voskuil, Y. Liu, J.A. Mangan, I.M. Monahan, G. Dolganov, B. Efron, P.D. Butcher, C. Nathan and others. 2003. Transcriptional adaptation of Mycobacterium tuberculosis within macrophages: insights into the phagosomal environment. J. Exp. Med. 198: 693-704.

Silberbach M., M. Schäfer, A.T. Hüser, J. Kalinowski, A. Pühler, R. Krämer and A. Burkovski. 2005. Adaptation of Corynebacterium glutamicum to ammonium limitation: a global analysis using transcriptome and proteome techniques. Appl. Environ. Microbiol. 71: 2391-2402.

Slayden R.A., D.L. Knudson and J.T. Belisle. 2006. Identification of cell cycle regulators in Mycobacterium tuberculosis by inhibition of septum formation and global transcriptional analysis. Microbiology 152: 1789-1797.

Sohaskey C.D. and L.G. Wayne. 2003. Role of narK2X and narGHJI in Hypoxic Upregulation of Nitrate Reduction by Mycobacterium tuberculosis. J. Bacteriol. 185: 7247-7256.

Tan M.P., P. Sequeira, W.W. Lin, W.Y. Phong, P. Cliff, et al. 2010. Nitrate respiration protects hypoxic Mycobacterium tuberculosis against acid- and reactive species stresses. PLoS ONE 5(10): e13356. doi: 10.1371/journal.pone.00133356.

Wang S., F. Liu and B. Zhao. 2000. Study on mRNA differential display in Mycobacterium tuberculosis H37Rv and H37Ra. Zhonghua Jie He He Hu Xi Za Zhi 23: 669-671.

Yang L.L., M.C. Wang, L.G. Chen and C.C. Wang. 2003. Cytotoxic activity of coumarins from the fruits of Cnidium monnieri on leukemia cell lines. Planta. Med. 69: 1091-1095.

Yu L., H. Xiang, J. Fan, D. Wang, F. Yang, N. Guo, Q. Jin and X. Deng. 2008. Global transcriptional response of Staphylococcus aureus to rhein, a natural plant product. J. Biotechnol. 135: 304-308. Yu L., N. Guo, R. Meng, B. Liu, X. Tang, J. Jin, Y. Cui and X. Deng. 2010. Allicin-induced global gene expression profile of Saccharomyces cerevisiae. Appl. Microbiol. Biotechnol. 88: 219-229.

Yu L., W. Zhang, L. Wang, J. Yang, T. Liu, J. Peng, W. Leng, L. Chen, R. Li, Q. Jin. 2007. Transcriptional profiles of the response to ketoconazole and amphotericin B in Trichophyton rubrum. Antimicrob. Agents Chemother. 51: 144-153. 